\title{
Differential phase-contrast microscopy at atomic resolution
}

\author{
Naoya Shibataa ${ }^{1,2 \star}$, Scott D. Findlay ${ }^{3}$, Yuji Kohno ${ }^{4}$, Hidetaka Sawada ${ }^{4}$, Yukihito Kondo ${ }^{4}$ \\ and Yuichi Ikuhara ${ }^{1,5}$
}

\begin{abstract}
Differential phase-contrast (DPC) imaging enhances the image contrast of weakly absorbing, low-atomic-number objects in optical and X-ray microscopy ${ }^{1-4}$. In transmission electron microscopy ${ }^{5}$, this same imaging mode can image magnetic fields in magnetic materials at medium resolution ${ }^{6,7}$. Atomicresolution imaging of electromagnetic fields, however, is still a major challenge. Here, we demonstrate atomic-resolution DPC imaging of crystals using aberration-corrected scanning transmission electron microscopy. The image contrast reflects the gradient of the electrostatic potential of the atoms; that is, the atomic electric field, which is found to be sensitive to the crystal ionicity. Both the mesoscopic polarization fields within each domain and the atomic-scale electric fields induced by the individual electric dipoles within each unit cell can be sensitively detected in ferroelectric $\mathrm{BaTiO}_{3}$. The realization of atomic-resolution DPC microscopy opens a new dimension of microscopy from crystalline materials through to biological molecules.
\end{abstract}

Phase-contrast imaging, whereby phase changes experienced by the incident probe during the interaction with the object are converted into image contrast, has been used in a wide range of microscopy techniques, such as optical, X-ray and electron microscopy. One key technique for resolving the structure of weakly interacting phase objects in optical and X-ray microscopy is so-called DPC imaging, where images are formed by subtracting intensities on detector segments diametrically opposed about the optical axis. This converts beam deflection, which relates closely to the gradient of the phase change through the specimen, into image contrast $^{1,5}$. DPC has also been much studied at low-to-medium resolution in scanning transmission electron microscopy (STEM), especially for the imaging of magnetic structures ${ }^{6,7}$. For samples scattering weakly enough to be regarded as phase objects, DPC images in STEM represent the gradient of the object potential (that is, the object electromagnetic field) taken in the direction of the diametrically opposed detector segments ${ }^{5,8-10}$. Applying DPC imaging to sub-ångström resolution STEM may enable the direct characterization of local electromagnetic fields at the level of single atoms. Recently, we have developed a segmented-type annular detector suitable for atomic-resolution STEM imaging ${ }^{11}$. In the present study, we demonstrate DPC STEM at atomic resolution and emphasize the contrast characteristics reflecting the atomicscale local electric fields.

Figure 1a,b shows the principle of image contrast formation assuming an isolated atom for the scattering object, in part for simplicity and in part to lead into our discussion of atomic-resolution imaging. With the probe a long way from the atom, the electron intensity detected by the two quadrant segments should be equal. When scanning the electron probe across the atom, the probe is deflected by the atomic electric field, which is generally dominated by the positive atomic nucleus. As shown in Fig. 1a and the intensity profiles of each segment in Fig. 1b, the attraction felt by an electron beam passing to the left of the atom tends to deflect it towards the detector on the right; the signal on the right side detector segment $(\mathrm{Y})$ is a maximum and the signal on the left side detector segment $(\mathrm{X})$ is a minimum when the electron probe is slightly to the left of the atomic position. Likewise, the signal on $\mathrm{X}$ is a maximum and the signal on $\mathrm{Y}$ is a minimum when the electron probe is slightly to the right of the atomic position. Subtracting the signals from the two detector segments $(\mathrm{X}-\mathrm{Y})$ produces the difference profile shown at the bottom of Fig. 1b. This profile has a node (zero crossing) at the atom location. The profile is antisymmetric about this point, reflecting the reversal of the electric field direction across the atom. Thus, the atomic-resolution DPC STEM image should reflect the projected electric field distribution in the vicinity of the atom. It is to be anticipated that a well-aligned crystal will show the same behaviour, provided it is sufficiently thin and/or weakly scattering that it can be regarded as a phase object. Care would be needed to interpret atomic-resolution images in DPC mode from more strongly scattering specimens.

For an experimental demonstration, we observe a $\mathrm{SrTiO}_{3}$ crystal along the [001] direction. Figure 1c shows the schematic of the orientation relationship between the $\mathrm{SrTiO}_{3}$ crystal and the detector segments labelled $\mathrm{X}$ and $\mathrm{Y}$, which are aligned parallel to the [110] direction of the $\mathrm{SrTiO}_{3}$ sample. The probe-forming aperture semiangle was set to be $23 \mathrm{mrad}$. The angular range from the optical axis of the detector segments was $15.3-30.6 \mathrm{mrad}$ and the azimuthal span was $90^{\circ}$. Figure $1 \mathrm{~d}$ shows the DPC STEM image and its intensity profile as projected over the entire vertical direction in the image. The simultaneous annular dark field (ADF) STEM image (angular range: $30.6-61.2 \mathrm{mrad}$ ) and its intensity profile are used for reference as the peaks in the ADF image have been well-established as an indicator of the true atomic positions ${ }^{12}$. It is found that the DPC image intensity profile behaves such that there are nodes (zero crossings) at the atom sites. The DPC signal is antisymmetric about these points. The form of the DPC signal is thus in excellent agreement with the intuitive model of Fig. 1a and $b$. As a check on this interpretation, the usual phase-contrast transmission electron microscopy procedure of matching to image simulations was used to establish such experimental parameters as defocus and thickness (see the Supplementary Information for

\footnotetext{
${ }^{1}$ Institute of Engineering Innovation, School of Engineering, The University of Tokyo, Yayoi 2-11-16, Bunkyo-ku, Tokyo 113-8656, Japan, ${ }^{2}$ Japan Science and Technology Agency, PRESTO, 4-1-8 Honcho Kawaguchi, Saitama 332-0012, Japan, ${ }^{3}$ School of Physics, Monash University, Victoria 3800, Australia, ${ }^{4} J E O L$ Ltd., 1-2, Musashino 3-chome Akishima, Tokyo 196-8558, Japan, ${ }^{5}$ Nanostructures Research Laboratory, Japan Fine Ceramic Center, 2-4-1 Mutsuno, Atsuta-ku, Nagoya 456-8587, Japan. ^e-mail: shibata@sigma.t.u-tokyo.ac.jp.
} 

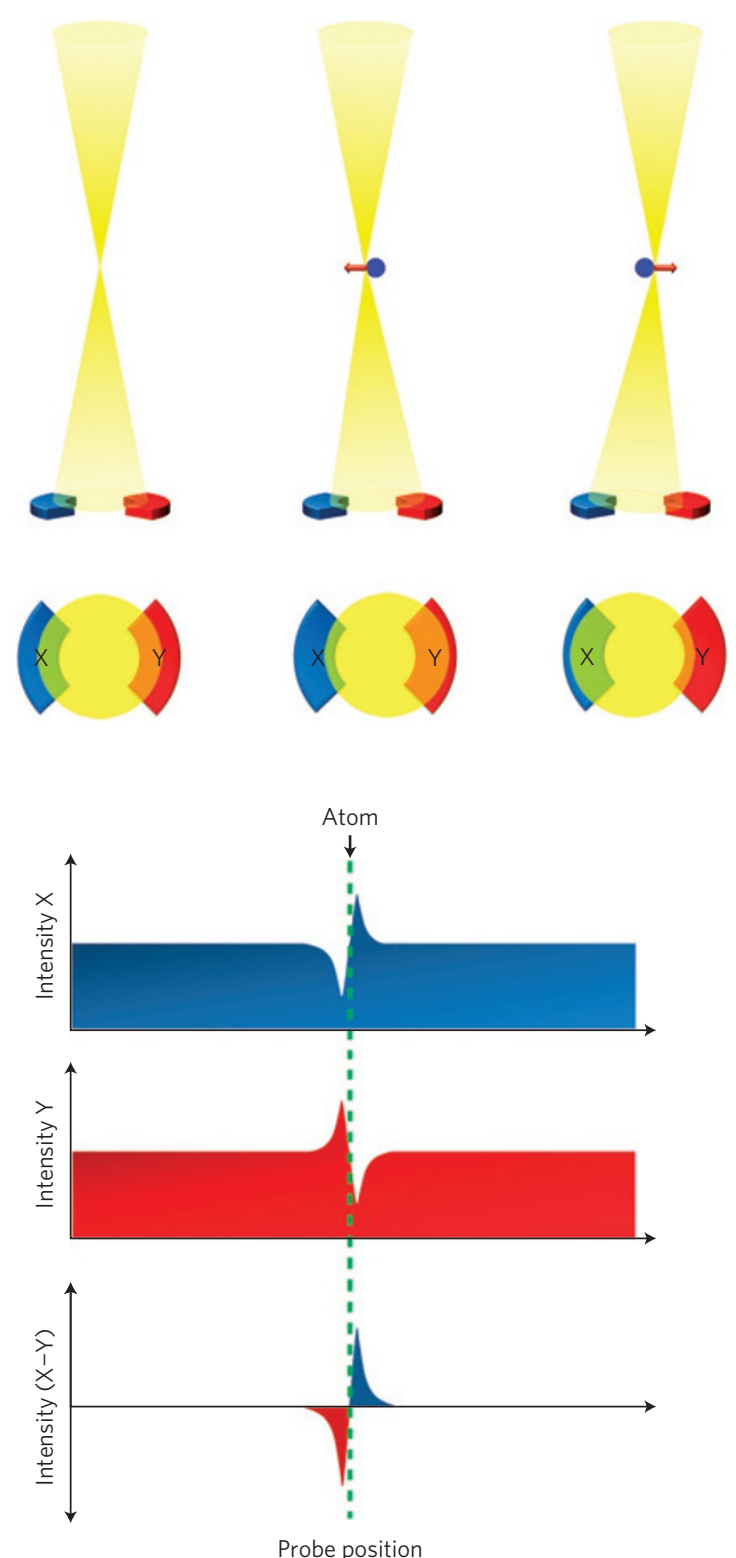

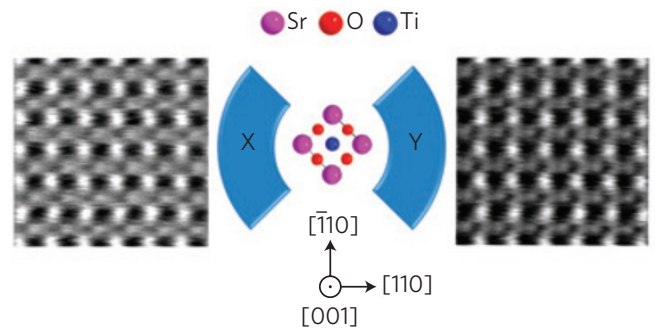

d
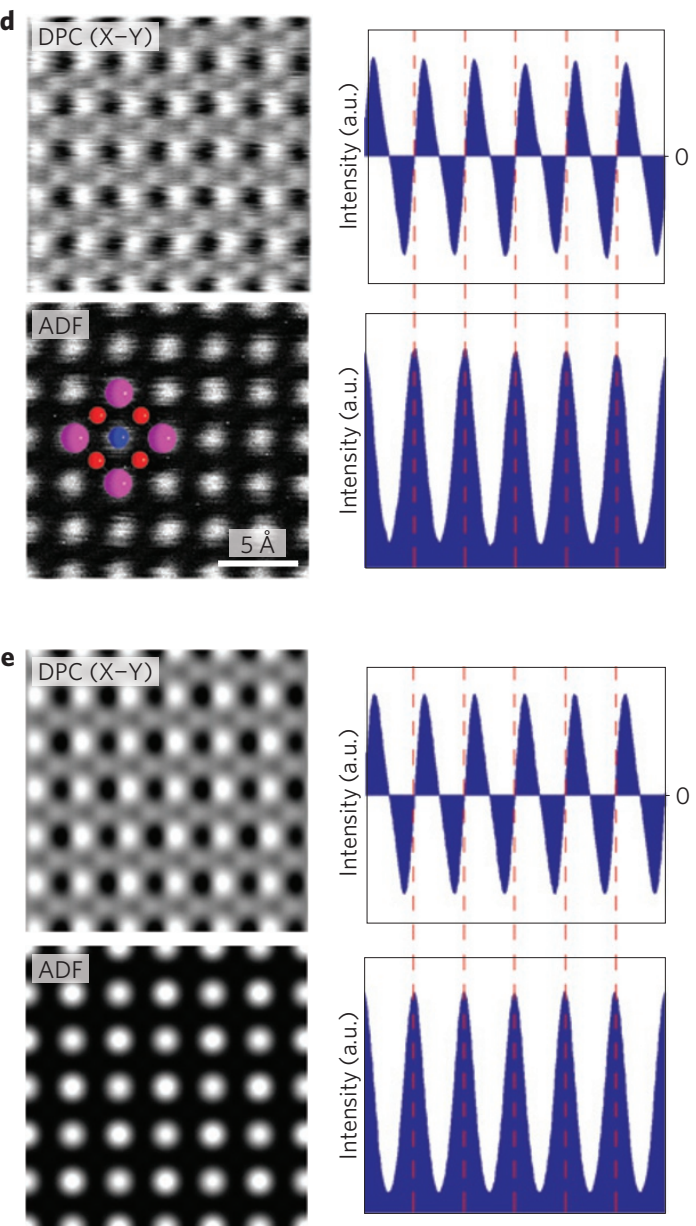

Figure 1 | Schematic showing the image formation mechanism of DPC STEM of a single atom and the experimental DPC STEM image of a SrTiO ${ }_{3}$ single crystal observed along the [001] direction. a, Schematic diagrams of the electron trajectory in the vicinity of the atom and the split electron detector segments, $X$ and $Y$. b, The image intensity profiles of each detector segment and a DPC (X-Y) image. The intensity profile of the DPC (X-Y) image, shown at the bottom of the figure, is antisymmetric about the zero crossing. c, Schematic showing the orientation relationship between the $\mathrm{SrTiO}_{3}$ single $\mathrm{crystal}$ and the quadrant annular detector segments used for the DPC STEM imaging. The angle range of the detector segments is set to be 15.3-30.6 mrad. Two simultaneous STEM images obtained by the two detector segments are also shown. These and all other images shown in this study are filtered by the radial difference filter ${ }^{20}$ released by the HREM Research, to subtract background noise. d, Experimental atomic-resolution DPC STEM image of the SrTiO 3 single crystal and its intensity profile across the horizontal direction. The simultaneously observed ADF STEM image and its intensity profile are also shown for reference. The angle range of the ADF detector is 30.6-61.2 mrad. e, Simulated atomic-resolution DPC STEM and ADF STEM images of the $\mathrm{SrTiO}_{3}$ single crystal and their image intensity profiles. The thickness $(t)$ and defocus $(\Delta f)$ values used for the image simulation were $t=3.1 \mathrm{~nm}$ and $\Delta f=-1.1 \mathrm{~nm}$, respectively.

details). We find the thickness to be consistent with the use of the phase object approximation. A simulated DPC STEM image and its intensity profile for $\mathrm{SrTiO}_{3}$ with the determined parameters are shown in Fig. 1e, and are clearly in good agreement with the experimental results.

The $\mathrm{SrTiO}_{3}$ structure is highly symmetric. This symmetry is broken in ferroelectric materials through the ferroelectric structural distortion and the concomitant change of the electric field. The electric field in ferroelectric materials may be considered to consist of two components: the uniform, long-range (mesoscopic) polarization field and another field which varies on the atomic scale $^{13}$. Having established that atomic-resolution DPC STEM imaging of crystalline materials is possible, we further demonstrate that it is sufficiently sensitive to detect the electric field anisotropy in ferroelectric materials. We use single-crystalline tetragonal $\mathrm{BaTiO}_{3}$, a prototypical perovskite ferroelectric. The ferroelectricity of $\mathrm{BaTiO}_{3}$ originates from the cooperative movement of ions within the unit cell. In the room-temperature ferroelectric tetragonal 

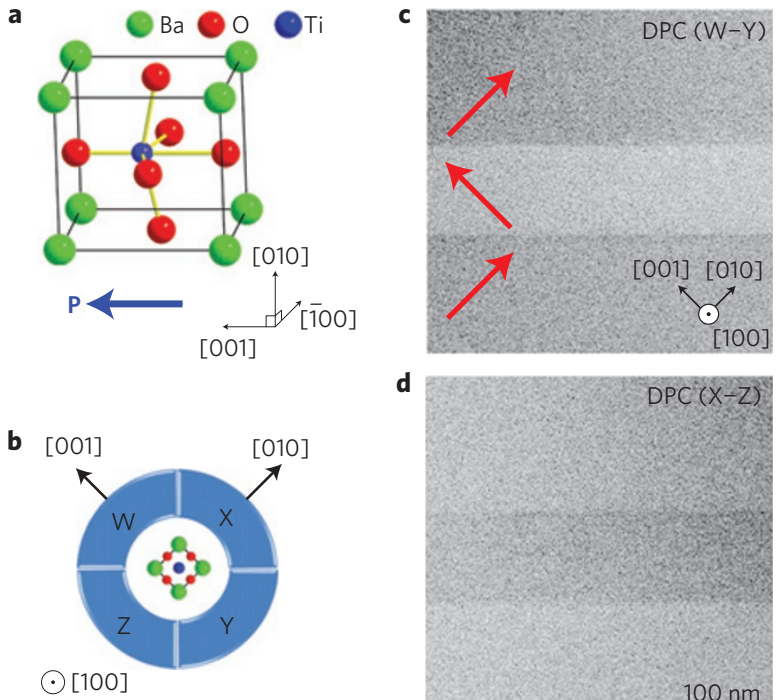

d

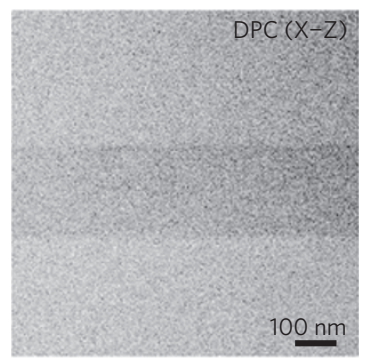

Figure 2 | DPC STEM images of domains in a tetragonal $\mathrm{BaTiO}_{3}$ crystal observed along the [100] direction. $\mathbf{a}$, Unit structure of tetragonal $\mathrm{BaTiO}_{3}$. b, Schematic showing the orientation relationship between the tetragonal $\mathrm{BaTiO}_{3}$ crystal and the quadrant annular detector segments used for DPC STEM imaging. c, Experimental DPC STEM image of (W-Y). The red arrows in the image indicate the direction of polarization in each domain gauged by the DPC STEM image intensities. $\mathbf{d}$, Experimental DPC STEM image of $(X-Z)$.

structure, the $\mathrm{Ba}$ and $\mathrm{Ti}$ sub-lattices are shifted relative to the oxygen sub-lattice, as shown schematically in Fig. 2a. This tetragonal distortion splits the centre of charges, resulting in the formation of a dipole moment $\mathbf{P}$ parallel to the $c$ axis. Figure $2 \mathrm{~b}$ shows the schematic of the orientation relationship between the $\mathrm{BaTiO}_{3}$ crystal and the quadrant annular detector, and Fig. $2 \mathrm{c}$ and d shows the simultaneous low-magnification DPC $(\mathrm{W}-\mathrm{Y})$ and $(\mathrm{X}-\mathrm{Z})$ images of the $90^{\circ}$ domain structure observed along the [100] direction. The domain structure is clearly visualized, with differences in image contrast due to the different orientation of the mesoscopic polarization fields in each domain. The image contrast variation in the two images can be consistently explained if the polarization directions are oriented as indicated by the red arrows. We also rotated the detector by $45^{\circ}$ and observed the same region (see the Supplementary Information). The contrast changes and relative intensities on the detector segments also confirm the proposed domain orientation. Thus DPC STEM can visualize the mesoscopic polarization fields in a ferroelectric crystal.

Quantification of the mesoscopic fields by DPC is the next step, and requires precise characterization of the specimen thickness and detector response. The process for quantifying the mesoscopic fields by DPC STEM is discussed in detail in the Supplementary Information, where it is shown that the deflection evidenced by the contrast variation in Fig. $2 \mathrm{c}$,d is much smaller than would be expected if the total field were equal to the bulk saturation polarization. This suggests that the polarization field is screened at surfaces and/or interfaces within the material.

Figure 3 shows atomic-resolution DPC STEM images inside a single domain of tetragonal $\mathrm{BaTiO}_{3}$. As schematically shown in Fig. 3a, the diametric direction of the two detector segments ( $\mathrm{W}$ and Y) was carefully oriented parallel to the [001] polarization direction. Figure $3 \mathrm{c}$ shows the atomic-resolution DPC (W-Y) and ADF STEM images observed along the [100] direction. The corresponding projected image intensity profiles across the $c$ axis direction are also shown. Here, the intensity profiles are projected across the entire width of the images. The atomic positions determined by the simultaneous ADF STEM image always lie at the nodes of the DPC STEM image intensity profile, characteristic of the atomic-resolution DPC STEM images. However, whereas the DPC intensity profiles across the $\mathrm{Ba}$ columns are nearly antisymmetric to the zero crossing, those of the Ti-O columns exhibit clear asymmetry. In contrast, the atomic-resolution DPC (X-Z) STEM image in Fig. 3d, with the diametrical direction of the segments oriented perpendicular to the [001] polarization direction, shows no asymmetric features in either the $\mathrm{Ba}$ or $\mathrm{Ti}-\mathrm{O}$ columns. This difference must relate to the polarization; the asymmetric profile is anticipated to indicate the zigzag distortion of the TiO column which arises from the displacement of the $\mathrm{Ti}$ ions relative to the $\mathrm{O}$ ions along these atomic columns in the polarization direction. Using analysis and simulation to further the interpretation, the images can tell us even more.

Figure $3 \mathrm{e}$, f show the simulated DPC (W-Y) and DPC (XZ) STEM images, the corresponding ADF image, and their projected image intensity profiles, based on the tetragonal $\mathrm{BaTiO}_{3}$ crystal structure determined by X-ray structure refinement ${ }^{14}$. The simulations are seen to be in good agreement with the experimental findings. Moreover, although the profile asymmetry in the atomic-resolution DPC (W-Y) signal derives from the zigzag distortion of the Ti-O columns along the [100] viewing direction, insomuch as distortion is a prerequisite for asymmetry, it was further necessary to use scattering factors for ions ${ }^{15}$ to obtain agreement with the experimental data. Figure $3 \mathrm{~b}$ shows the comparison between simulated DPC (W-Y) profiles of a tetragonal $\mathrm{BaTiO}_{3}$ crystal using neutral atom (left panel) and ionic (right panel) potentials (see Supplementary Information for details). The use of scattering factors for neutral atoms is unable to reproduce the large profile asymmetry seen in the experimental images. This discrimination of the ionicity (charge redistribution) shows just how sensitive the DPC technique is to the atomic-scale electric fields. The combination of structural distortion and ionicity detected here are precisely the factors which indicate the electric dipole formation in the unit cell. This sensitivity, not just to the structural distortion but also to the electric fields via the sensitivity to charge state, provides additional information on the polarization of the material to that from techniques that probe the structural distortion alone $e^{16,17}$. These previous studies ${ }^{16,17}$ measure column shifts but can only estimate spontaneous polarization based on Born effective charge analysis. Our approach can combine the two styles of analysis: the new segmented-type annular detector provides a wealth of signals from which both shifts of light-element and heavy-element columns and also the total electric field can be measured directly and simultaneously. It is important to appreciate that the localized asymmetry in the atomic-resolution DPC profile is distinct from the effect of the mesoscopic polarization field, which is by definition uniform within a given domain. A constant field implies a linear potential gradient, which in DPC corresponds to a constant vertical offset in the DPC profile. The detector is not sufficiently well characterized to provide a quantitative measure of this offset at atomic resolution, which will be difficult to discriminate from the consequences of slight detector misalignment. It was distinguishable at low magnification because the sample was thicker, enhancing the effect, and because it sufficed to see the relative difference between different domains. But, with further refinements in the detector system, atomic-resolution measurement of the mesoscopic field should be possible.

In summary, we have demonstrated that DPC imaging at atomic resolution is possible in aberration-corrected STEM. The ability to detect very localized fields will assist our fundamental understanding of many ferroic and polar materials, where the correlation between local atomic-scale structures and resultant electromagnetic fields is the key to understanding their extraordinary properties. 


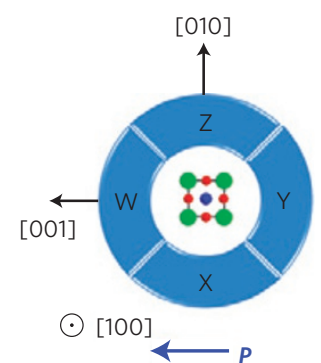

b

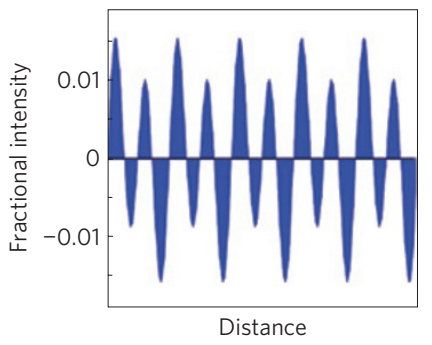

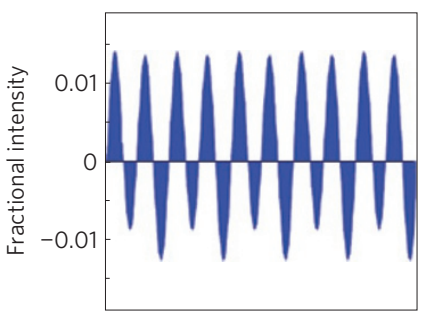

Distance
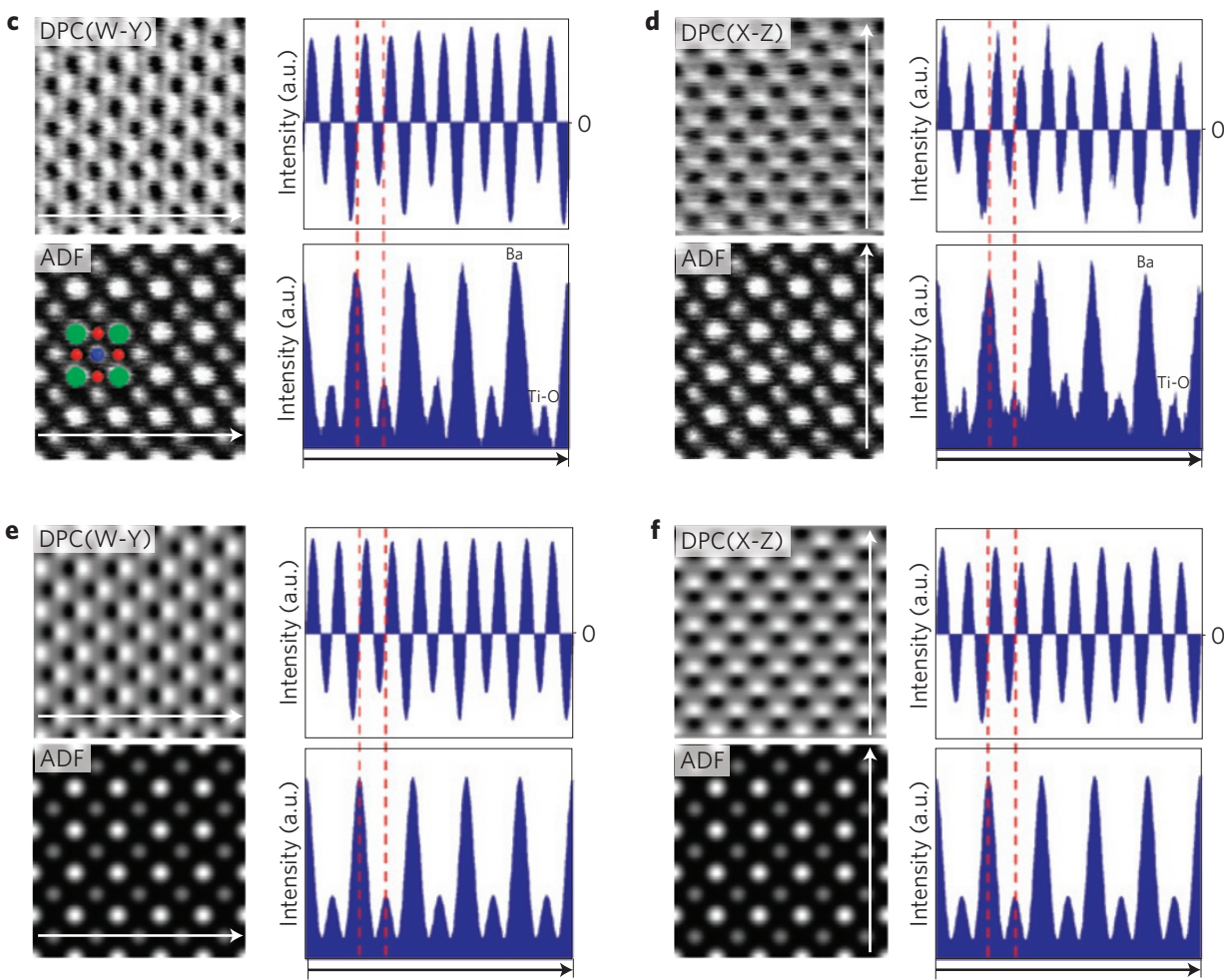

Figure 3 | Atomic-resolution DPC STEM images of a tetragonal $\mathrm{BaTiO}_{3}$ crystal observed along the [100] direction. $\mathbf{a}$, Schematic showing the orientation relationship between the tetragonal $\mathrm{BaTiO}_{3}$ crystal and the quadrant annular detector segments used for DPC STEM imaging. The diagonal direction of the two detector segments ( $W$ and $Y$ ) is oriented parallel to the [001] direction, whereas that of the detector segments (X and $Z$ ) is oriented parallel to the [010] direction. The angle range of the detector segments is set to be 15.3-30.6 mrad. b, Simulated DPC (W-Y) image intensity profiles of a tetragonal $\mathrm{BaTiO}_{3}$ crystal using neutral atom (left panel) and ionic (right panel) scattering factors. In these simulations, all the parameters, such as atomic coordinates, microscope condition, detector size and specimen thickness are the same as in e and $\mathbf{f}$, as given below. c,d, Experimental DPC STEM images and corresponding projected intensity profiles. c shows the DPC STEM image of (W-Y) and $\mathbf{d}$ shows the DPC STEM image of (X-Z). The simultaneous ADF STEM images and corresponding image intensity profiles are also shown. The image intensity profile of the DPC (X-Z) image seems to be noisier than that of the DPC (W-Y) image because the scan noise is perpendicular to the direction over which we project to extract the intensity profile of the DPC $(X-Z)$ image rather than parallel to it, as is the case for the DPC $(W-Y)$ image. e,f, Simulated DPC STEM images and corresponding image intensity profiles using ionic scattering factors. e shows the simulated DPC STEM image of (W-Y) and $\mathbf{f}$ shows the simulated DPC STEM image of (X-Z). In c-f the profile is taken along the direction indicated by the arrows after projection over the direction perpendicular to the arrows. The red dotted lines highlight the alignment of features between the DPC and ADF intensity profiles. The thickness and defocus values used for the image simulation were $t=1.6 \mathrm{~nm}$ and $\Delta f=0 \mathrm{~nm}$, respectively.

\section{Methods}

Commercially available $\mathrm{SrTiO}_{3}$ and $\mathrm{BaTiO}_{3}$ single crystal substrates were used as a starting material (Furuuchi Chemical). The substrates were cut and mechanically polished with diamond suspension to have a total thickness of less than $100 \mu \mathrm{m}$. The samples were then dimpled using a dimple grinder to obtain a thin area at the centre of the sample. To obtain electron transparency, we used a standard Ar ion-beam thinning method. The accelerating voltage was gradually decreased as thinning progressed to minimize surface damage and contamination. STEM images were taken with a $200 \mathrm{kV}$ JEM-2100F TEM/STEM electron microscope (JEOL) equipped with an aberration corrector (CEOS $\mathrm{GmbH}$ ). The instrumentation of the segmented annular all-field detector used in this study is reported elsewhere ${ }^{11}$

The image simulations used the multislice frozen phonon model ${ }^{18,19}$ based on the elastic structure factors for ions ${ }^{15}$. X-ray structure refinement results were used for the polarized $\mathrm{BaTiO}_{3}$ structure ${ }^{14}$. To include the long-range field due to polarization, the $\mathrm{BaTiO}_{3}$ unit was tiled seven times in one direction but placed within a supercell of length thirteen times the single cell repeat distance. The superposition of the potentials for the isolated ions at locations as per the polarized structure then naturally produces a linear potential within the crystal, describing the long-range polarization field. This tails off in the vacuum gap in the supercell such that periodicity of the supercell, implicit in the calculations through the use of Fourier transforms in the multislice algorithm, is maintained. Similar to the findings in previous work ${ }^{14}$, the polarization field thus produced differs in magnitude from that measured experimentally. As discussed in the main text, the effect of the long-range polarization field on the electron beam is sufficiently small for our sample that the discrepancy should not affect our results. The microscope parameters used are based on the experimental values. A $200 \mathrm{keV}$, aberration-free (excepting defocus) probe with probe-forming aperture semiangle of $23 \mathrm{mrad}$ was 
assumed. The detector angles and orientation relative to the sample were as per the experiment. As discussed in the Supplementary Information, thickness and defocus values were determined via systematic matching with the experimental data from multiple detectors.

\section{Received 3 February 2012; accepted 7 May 2012; published online} 24 June 2012

\section{References}

1. Hamilton, D. K. \& Sheppard, C. J. R. Differential phase contrast in scanning optical microscopy. J. Microsc. 133, 27-39 (1984).

2. Pfeiffer, F., Weitkamp, T., Bunk, O. \& David, C. Phase retrieval and differential phase-contrast imaging with low-brilliance X-ray sources. Nature Phys. 2, 258-261 (2006).

3. Amos, W. B., Reichelt, S., Cattermole, D. M. \& Laufer, J. Re-evaluation of differential phase contrast (DPC) in a scanning laser microscope using a spit detector as an alternative to differential interference contrast (DIC) optics. J. Microsc. 210, 166-175 (2003).

4. Hornberger, B. et al. Differential phase contrast with a segmented detector in a scanning X-ray microscope. J. Synchrotron Radiat. 15, 355-362 (2008).

5. Dekkers, N. H. \& de Lang, H. Differential phase contrast in a STEM. Optik 41, 452-456 (1974).

6. Chapman, J. N. The investigation of magnetic domain structures in thin foils by electron microscopy. J. Phys. D 17, 623-647 (1984).

7. Chapman, J. N., McFadyen, I. R. \& McVitie, S. Modified differential phase contrast Lorentz microscopy for improved imaging of magnetic structures. IEEE Trans. Magn. 26, 1506-1511 (1990).

8. Rose, H. Nonstandard imaging methods in electron microscopy. Ultramicroscopy 2, 251-267 (1977).

9. Stewart, W. C. On differential phase contrast with an extended illumination source. J. Opt. Soc. Am. 66, 813-818 (1976).

10. Cowley, J. M. Configured detectors for STEM imaging of thin specimens. Ultramicroscopy 49, 4-13 (1993).

11. Shibata, N. et al. New area detector for atomic-resolution scanning transmission electron microscopy. J. Electron Microsc. 59, 473-479 (2010).

12. Pennycook, S. J. \& Jesson, D. E. High-resolution incoherent imaging of crystals. Phys. Rev. Lett. 64, 938-941 (1990).

13. Lichte, H., Reibold, M., Brand, K. \& Lehmann, M. Ferroelectric electron holography. Ultramicroscopy 93, 199-212 (2002).
14. Rother, A. et al. X-ray investigation, high-resolution electron holography, and density functional calculations of single-crystalline $\mathrm{BaTiO}_{3}$. Phys. Rev. B. 74, 134116 (2006).

15. Peng, L-M. Electron scattering factors of ions and their parameterization. Acta Crystallogr. A 54, 481-485 (1998).

16. Jia, C-L. et al. Atomic-scale study of electric dipoles near charged and uncharged domain walls in ferroelectric films. Nature Mater. 7, 57-61 (2008).

17. Borisevich, A. Y.et al. Mapping octahedral tilts and polarization across a domain wall in $\mathrm{BiFeO}_{3}$ from Z-contrast scanning transmission electron microscopy image atomic column shape analysis. ACS Nano 4, 6071-6079 (2010).

18. Loane, R. F., Xu, P. \& Silcox, J. Thermal vibrations in convergent-beam electron-diffraction. Acta Crystallogr. A 47, 267-278 (1991).

19. Kirkland, E. J. Advanced Computing in Electron Microscopy (Plenum, 1998).

20. Kilaas, R. Optimal and near-optimal filters in high-resolution electron microscopy. J. Microsc. 190, 45-51 (1998).

\section{Acknowledgements}

We thank N. Saito for assistance in STEM specimen preparation. This work was supported by the PRESTO, Japan Science and Technology Agency. A part of this work was also supported by the Japan Society for the Promotion of Science through its 'Funding Program for World-Leading Innovative R\&D on Science and Technology (FIRST Program)'. A part of this work was conducted in the Research Hub for Advanced Nano Characterization, The University of Tokyo, supported by MEXT, Japan. N.S. also acknowledges support from the Industrial Technology Research Grant Program in 2007 from the New Energy and Industrial Technology Development Organization and the Grant-in-Aid for Young Scientists (A) $(23,686,093)$ from MEXT. S.D.F. acknowledges support by the Australian Research Council.

\section{Author contributions}

N.S. and S.D.F. designed the study and wrote the paper. N.S. performed the STEM experiments and S.D.F. performed image simulations. Y.Kohno, H.S. and Y.Kondo supported the experiments. Y.I. contributed the discussion and suggestions.

\section{Additional information}

The authors declare no competing financial interests. Supplementary information accompanies this paper on www.nature.com/naturephysics. Reprints and permissions information is available online at www.nature.com/reprints. Correspondence and requests for materials should be addressed to N.S. 